\title{
Preguntas a la coyuntura. Reflexiones, problemas y preocupaciones acerca del trabajo docente, el uso de las tecnologías y las desigualdades ante la pandemia
}

Questions to the conjuncture. Reflections, problems and concerns about teaching work, the use of technologies and inequalities in the face of the pandemic

\author{
Yamile Socolovsky \\ https://orcid.org/0000-0002-8358-1853 \\ yamilesocolovsky@gmail.com \\ Secretaria de Relaciones Internacionales I Directora \\ del Instituto de Estudios y Capacitación I CONADU
}

Me propongo desarrollar una breve reflexión en torno al tema de las condiciones del trabajo docente en las universidades, a partir de varias preocupaciones que cobran especial urgencia y visibilidad en el actual contexto generado por la pandemia del Covid-19, para intentar ponerlas en la perspectiva de ciertos debates previos que organizan una mirada crítica sobre la universidad. Esta perspectiva asume su compromiso inicial con una concepción política que entiende que la universidad debe constituirse como un territorio de derechos. Porque la educación es en general, y también en el nivel superior, un derecho humano universal, como lo sostuviera la Declaración de la Conferencia Regional de Educación Superior en 2008 y tal como se reafirmó en la última CRES, realizada en junio de 2018, en coincidencia con la celebración del Centenario de la Reforma Universitaria de Córdoba. Y porque, al mismo tiempo, el conjunto de la actividad que se desarroIla en las instituciones que conforman el sistema universitario público encuentra su sentido y legitimación en su vocación y capacidad de ofrecer al conjunto de nuestro pueblo un ámbito en el cual la enseñanza, la formación de profesionales, la producción de conocimiento, la circulación de saberes, la restitución de memorias colectivas, la contribución al debate democrático y las numerosas tareas que en ellas pueden realizarse y promoverse, se orienten a la construcción de una sociedad más igualitaria, más respetuosa de las diversidades, más ca- 
paz de asegurar una vida digna para sus integrantes, en un mundo más habitable. Es en este sentido que sostenemos que la universidad es un derecho colectivo de nuestro pueblo. En esta perspectiva entendemos, además, que la actividad académica que llamamos docencia universitaria (y que trasciende, pero a la vez mantiene en su centro, la función de la enseñanza) es en sentido propio un trabajo, y que, por lo tanto las condiciones adecuadas para su realización y protección deben ser también reconocidas como derechos fundamentales de las personas. En estos términos, la universidad en tanto que "territorio de derechos" debe ser concebida de manera integral, como un ámbito en el cual la vigencia de los derechos laborales y sindicales sus trabajadoras y trabajadores no puede escindirse de la promoción y garantía del derecho a la educación, al conocimiento y la cultura que es responsabilidad de las instituciones asegurar.

\section{LA DOCENCIA UNIVERSITARIA EN EL CONTEXTO DE LA PANDEMIA}

A partir del establecimiento del Aislamiento Social Preventivo y Obligatorio (ASPO) en nuestro país, como medida necesaria para afrontar la pandemia producida por el Covid-19 limitando la velocidad del avance de los contagios, y adecuando la capacidad del sistema de salud a la emergencia sanitaria, la suspensión o reducción de todas las actividades que implican reunión y movilización de personas ha impactado severamente sobre el conjunto de las y los trabajadores de todos los sectores, y muy especialmente sobre quienes carecen de los reaseguros asociados al empleo formal. Es fundamental no perder de vista que las consecuencias de la paralización de numerosas actividades económicas representan efectos muy gravosos para una población económicamente activa hoy conformada en algo más del $50 \%$ por trabajadoras y trabajadores informales o carentes de registración: desempleo, pérdida de ingresos, y ausencia de prestaciones y de garantías que en estas circunstancias son aún más necesarias que antes, como los servicios de salud, o las licencias remuneradas. Dicho esto, y aún cuando en términos generales la docencia universitaria goza, en el sistema público argentino, de una situación laboral relativamente segura, respaldada por un Convenio Colectivo de Trabajo que establece un importante piso de derechos para el sector, es necesario señalar que, en sus propios términos, la coyuntura reviste también una complejidad que es preciso atender. No solamente porque no toda la docencia en nuestras universidades se encuentra actualmente en condiciones de contratación regulares, y persisten en el sistema formas precarias de empleo que exponen a quienes desarrollan su actividad bajo estas modalidades a sufrir mayores arbitrariedades y abusos, sino porque, además, se han producido cambios forzados, ge- 
neralizados y muy significativos en la forma de trabajo, que están generando fuertes malestares, dificultades y preocupaciones sobre las cuales vamos a detenernos un poco más adelante.

Al igual que en los niveles obligatorios del sistema educativo, la decisión de suspender las actividades presenciales, en el marco de las medidas generales de distanciamiento físico, sin que ello comportara una interrupción del dictado de clases, produjo un abrupto traslado de las acciones de enseñanza al terreno virtual. Ante todo, es preciso comprender que no se trata de la mera "virtualización" de las clases, sino de la realización de la actividad de enseñanza y aprendizaje a distancia, en el contexto del distanciamiento físico que exige la pandemia.

\section{Ante todo, es preciso comprender que no se trata de la mera "virtualización" de las clases, sino de la realización de la activi- dad de enseñanza y aprendizaje a distancia, en el contexto del distanciamiento físico que exige la pandemia.}

Esto implica que no sólo es una exigencia que obligó a docentes, estudiantes, cátedras, e instituciones a apurar una reprogramación de actividades, a trasladar al entorno virtual propuestas pedagógicas concebidas para la presencialidad, y a adquirir velozmente las destrezas mínimas necesarias para emplear las tecnologías disponibles, sino a hacerlo con el equipamiento y la conectividad con la que cada quien contaba en su domicilio, y cuyo uso, en muchos casos, debe ser compartido con otras personas. La "cuarentena" exige, además, para quienes tienen personas a cargo, tareas de cuidado adicionales, que la suspensión de la presencialidad en todos los niveles educativos torna especialmente complejas para quienes deben estudiar o trabajar en su domicilio, y muy particularmente para las mujeres, en virtud de los roles impuestos por la cultura patriarcal. A la sobrecarga por tareas de cuidado de hijas e hijos hay que añadir, frecuentemente, la asistencia a otras personas que requieren cuidados, especialmente mayores dependientes que a veces viven en otros domicilios, y cuya asistencia exige desplazamientos que las restricciones a la movilidad y los recaudos sanitarios y de higiene tornan un factor adicional de cansancio y estrés. En este sentido, señalamos también una situación de invasión de la privacidad, a través de esta forma del trabajo, que es un problema difícil de resolver, mucho más difícil cuando la situación se produce en este contexto generalizado de aislamiento. Es preciso observar, además, y no perder de vista, que todas las dificultades que han debido afrontar las y los docentes aquejan también a sus estudiantes, en muchas ocasiones incluso con mayor intensidad. Finalmente, es necesario también subrayar el hecho de que hablamos del distanciamiento que impone la pandemia, porque ello supone un componente de angustia e incertidumbre que no puede ser ignorado en la consideración de las condiciones en las que se está desarrollando, en estas horas, la enseñanza y el aprendizaje en las 
universidades. La incertidumbre se extiende a todas las dimensiones de nuestra vida, en términos personales y colectivos, y es parte de la excepcionalidad de la situación general que hemos tratado de caracterizar. Sin embargo, en algunos aspectos, se hace necesario, y es posible, reducirla para que no genere un malestar evitable, para que no alimente situaciones injustas, y para que esa misma indeterminación no conspire contra el propósito de sostener actividades socialmente valiosas como, en este caso, la educación.

\section{¿QUÉ NOS PREOCUPA EN ESTE CONTEXTO?}

Si bien las preocupaciones son muchas, quisiera detenerme en tres grandes cuestiones. En primer lugar, la experiencia generalizada de una sobrecarga de trabajo mucho más pesada que la habitual.

Como hemos adelantado en nuestra caracterización anterior, el modo en que se produce la adecuación de la docencia a la modalidad del trabajo remoto realizado en domicilio (que comienza a llamarse genéricamente "teletrabajo") ha generado un importante incremento y complejización de las tareas, y ha favorecido no pocas situaciones de abuso en las instituciones y en las cátedras o áreas en las que se organiza normalmente el trabajo docente. Esta constituye una primera preocupación, inmediata y más visiblemente ligada a las condiciones de trabajo. Las y los docentes necesitamos, en estas circunstancias, mucho más tiempo y esfuerzo para la preparación y el mal llamado "dictado" de clases, para acompañar a les estudiantes y lograr atender también a sus dificultades, para redefinir nuestros objetivos y encontrar formas razonables de evaluar lo realizado en condiciones tan excepcionales y para acreditarlo en algún formato compatible con las exigencias usuales de las instituciones.

\section{Las y los docentes necesitamos, en estas circunstancias, mucho más tiempo y esfuerzo para la preparación y el mal Ilamado "dictado" de clases, para acompañar a les estudiantes y lograr atender también a sus dificultades, para redefinir nuestros objetivos y encontrar formas razonables de evaluar lo reali- zado en condiciones tan excepcionales y para acreditarlo en algún formato compatible con las exigencias usuales de las instituciones.}

Las dificultades actuales no se reducen a la mayor cantidad de tiempo necesario para llevar a cabo aquellas tareas, sino que se refieren también a la organización del tiempo y la distribución de las tareas. Por una parte, el trabajo en domicilio genera una notoria dificultad para separar el tiempo destinado al trabajo y el que demandan, especialmente para las mujeres, los cuidados y el trabajo reproductivo en 
general, pero también el que se necesita para descansar, o para realizar otras actividades importantes y necesarias para una buena vida. Por otra parte, la asignación de tareas en el cumplimiento de la propia función de enseñanza se torna difusa, y en no pocas ocasiones la continuidad de la acción educativa se basa en una delegación de responsabilidades que exceden los términos en que se definen los roles correspondientes a las categorías que conforman los equipos docentes, con una clara sobreexigencia para quienes ocupan posiciones auxiliares, que son normalmente, además, quienes tienen el contacto más directo y constante con las y los estudiantes.

Estas cuestiones tienen claramente una relación con el conjunto de la situación que configura el aislamiento, pero también es importante pensar: ¿Qué pasa con la educación a distancia o con esta forma particular del trabajo remoto que constituye la educación a distancia? ¿La educación a distancia demanda más o menos tiempo y esfuerzo que la educación presencial? Lo cierto es que no lo sabemos, porque uno de los problemas que observamos es que a la mayor parte de las y los docentes de nuestras universidades, como las y los docentes del conjunto del sistema educativo nos falta preparación para desarrollar nuestras tareas en contextos virtuales. Entonces, un problema común frente al uso de las nuevas tecnologías se vincula con la falta de preparación para desarrollar nuestro hacer docente a través de la mediación tecnológica que en este momento se nos plantea como la única forma de sostener la continuidad pedagógica.

Ligado al problema que genera el uso de estas nuevas tecnologías, se visibiliza una dificultad que particularmente las mujeres conocemos bien y que tal vez ahora, otros comiencen a conocer mejor, que es la dificultad de conciliar el tiempo del trabajo con el tiempo que se destina a los cuidados en la familia, al descanso, a la recreación, a otras actividades. La desigual distribución de las responsabilidades del cuidado, y la ausencia de una política que proporcione una respuesta colectiva a un problema social, es uno de los factores más determinantes de las dificultades que encuentran las mujeres para una participación plena e igualitaria en el mundo del trabajo en general, y en la docencia universitaria y la actividad académica en particular. Al parecer, la pandemia está haciendo más visible un problema largamente ignorado, y es, en ese sentido, una oportunidad para que esa demanda cobre la densidad necesaria para empezar a lograr respuestas, también en el plano de las políticas institucionales y de la regulación del trabajo.

La desigual distribución de las responsabilidades del cuidado, y la ausencia de una política que proporcione una respuesta colectiva a un problema social, es uno de los factores más determinantes de las dificultades que encuentran las mujeres para una participación plena e igualitaria en el mundo del trabajo en general, y en la docencia universitaria y la actividad académica en particular. 
Además, es una situación que agudiza el problema de la distribución de las tareas y de la organización del trabajo docente. Es necesario, más allá de esta coyuntura, repensar y discutir la cultura jerárquica que organiza el trabajo en las cátedras y, en general, la configuración de nuestro sistema universitario, caracterizado por un predominio de las dedicaciones simples en la base de la pirámide que es la forma normal de las plantas docentes. Si las dedicaciones simples son más frecuentes entre los y las auxiliares, aquellos y aquellas docentes que tienen el trabajo más directo y continuo con el estudiantado, y, además, se asume que destinan una proporción mayor de su dedicación horaria a las actividades de enseñanza, esta distribución de responsabilidades, que ya era fuente de malestares en la presencialidad, no deja de agudizarse en esta situación.

Frente a este conjunto de problemas, es evidentemente necesario construir y de establecer regulaciones para la situación de emergencia, pero también es oportuno debatir y comenzar a producir reaseguros permanentes para el futuro. Porque aún si no fuera a generalizarse esta modalidad de la educación a distancia, lo cierto es que la educación a distancia ya existía y estaba desarrollándose en nuestras universidades. No es necesario prever escenarios apocalípticos, con una completa sustitución de la enseñanza presencial por la modalidad a distancia, para comprender que es perentorio construir aún mejores regulaciones para garantizar condiciones de trabajo adecuadas y, en ese contexto, volver a revisar las formas en que se organiza el trabajo docente, cómo se conforman y estructuran las plantas docentes, cómo se definen y se preservan las funciones propias de cada categoría, y cómo se proporciona la formación que nuestra actividad requiere también como un elemento imprescindible para su mejor ejercicio.

\section{LA PREGUNTA POR EL ROL DOCENTE Y LA MEDIACIÓN TECNOLÓGICA}

Aparece, también en estos días, una preocupación por la posibilidad de que esta abrupta incorporación de las tecnologías en la actividad de enseñanza pudiera implicar algo así como un borramiento de la figura docente. Esa es una preocupación que efectivamente no es menor, porque si bien las tecnologías son o están planteadas como mediadoras de esta actividad, es real que existen o hay ciertos usos de las tecnologías que permiten o favorecen un desplazamiento o una sustitución de la actividad docente por formas o dispositivos de administración de contenidos, de gestión de información, que desplazan la figura docente, que niegan finalmente el hecho docente mismo y que son funcionales a un proceso de mercantilización de la educación, que no es tan visible en nuestro país, pero que está desarrollándose con mucha fuerza, una fuerza muy preocupante en otros países, más aún en los niveles básicos de la educación que en la educación superior, problema que nos preocupa.

Al mismo tiempo, tenemos hoy una cantidad muy importante de docentes que en esta situación vuelven a preguntarse, y en algunos casos se preguntan por primera vez, cómo hacer para enseñar. Esta es una pregunta que, hay que decir, no es tan frecuente como quisiéramos en 
el mundo universitario. Seguramente no es así para quienes estamos discutiendo las cuestiones docentes, las cuestiones de la enseñanza, porque eso forma parte de nuestra actividad y nuestros intereses. Es una pregunta que parece básica, pero hay que pensar cómo se constituye en términos generales el mundo de la docencia universitaria, en el que suele asumirse que la formación profesional o académica en las disciplinas capacita por sí misma para su enseñanza, lo cual es un error que oculta un prejuicio despreciativo hacia la docencia y el desconocimiento de la pedagogía como un campo de estudios específico y necesario. Entonces, en algún sentido finalmente es positivo que una situación crítica nos haga preguntarnos cómo se enseña, cuáles son los objetivos de la enseñanza, cómo disponemos los recursos para la enseñanza. Esto es: cómo construimos estrategias para lograr esos objetivos, qué podría ser un recurso para la enseñanza, a quién le enseñamos o con quién construimos esa relación pedagógica, independientemente del territorio, virtual o presencial, en el que se despliega esa actividad.

Tenemos el desafío de aprovechar la coyuntura como una oportunidad para volver a pensar la relación pedagógica, y para descubrirla como un territorio en el que se construye comunicación, como una vinculación en la que se produce conocimiento. Pensar la cátedra como espacio de formación y circulación de saberes, para volver a considerar la importancia que tiene, que sigue teniendo, abordar una tarea pendiente en nuestras universidades como es el desarrollo de una pedagogía universitaria crítica, que no sólo piense la docencia en general, sino la docencia en la universidad. Detenernos a considerar cuáles son los modos, los lugares, en los que se produce conocimiento, quiénes son les sujetes entre quienes se produce el conocimiento, para seguir buscando las formas de superar la brecha entre la enseñanza y la investigación en las universidades y recuperar una perspectiva más integral de nuestra propia tarea.

Tenemos el desafío de aprovechar la coyuntura como una oportunidad para volver a pensar la relación pedagógica, y para descubrirla como un territorio en el que se construye comunicación, como una vinculación en la que se produce conocimiento. Pensar la cátedra como espacio de formación y circulación de saberes, para volver a considerar la importancia que tiene, que sigue teniendo, abordar una tarea pendiente en nuestras universidades como es el desarrollo de una pedagogía universitaria crítica, que no sólo piense la docencia en general, sino la docencia en la universidad.

Finalmente, cuando comprendemos la relación pedagógica como un lugar de producción de conocimiento, también es importante decir que la universidad no solo usa las tecnologías para la enseñanza, sino que además en ella se producen permanentemente tecnologías que procuran auxiliar tareas y resolver problemas en un abanico muy 
amplio de cuestiones. Y, que tal vez también sea parte de nuestra tarea docente -entendiendo la docencia como lugar de producción de conocimiento- pensar, por una parte cómo podemos apropiarnos críticamente de las tecnologías disponibles para la educación, pero sobre todo, cómo podemos producir las tecnologías que necesitamos para facilitar el desarrollo de la enseñanza que queremos. La única forma en que podemos asegurarnos de que la tecnología no sustituya, no desplace, no someta a quien desempeña el rol docente, es logrando que esa tecnología - que al fin y al cabo es producto de la inteligencia humana- sea nuestra.

\section{DESIGUALDADES, DERECHOS Y EMERGENCIA}

Pero sin dudas la mayor preocupación, presente en todas aquellas cuestiones, es la desigualdad, las desigualdades que pone a la vista la situación de la pandemia. Desigualdades materiales y simbólicas, desigualdades socio-económicas, laborales, habitacionales, de género, educativas; desigualdades en la situación de les estudiantes, en las condiciones de trabajo, y también aquellas que estructuran internamente y en todos sus niveles el propio sistema universitario. ¿El mundo, y también nuestro mundo educativo, nuestra universidad, va a ser más desigual después de la pandemia? Nuestra universidad, nuestra sociedad, nuestro país va a ser más desigual a menos que hagamos algo, en la emergencia, pero también -en esta emergencia- en relación a las condiciones estructurales que producen y reproducen esa desigualdad.

Entonces, junto con la reflexión por las condiciones del trabajo docente, es necesario volver a preguntarse qué se necesita hoy, en la universidad y de la universidad, para asegurar nuestros derechos, nuestro derecho a la educación, nuestro derecho al trabajo docente como un trabajo digno, y nuestro derecho, como pueblo, a ser protagonistas del proceso colectivo de producción, apropiación y disfrute del conocimiento y la cultura que hagan mejor la vida. En definitiva, qué se necesita hoy de la Universidad para que la pandemia no nos conduzca a un futuro más desigual y más injusto sino a un mundo, tal vez mejor, más solidario y más habitable para todos y todas. En ese sentido, aquella definición política que daba marco inicial a nuestras reflexiones, la reivindicación de la universidad como un derecho colectivo y como un factor decisivo para el desarrollo de una sociedad más justa, debe ser el principio rector de una mirada crítica que no deje de advertir, en la emergencia, las marcas de una inmensa tarea pendiente que no admite demoras.

*Este trabajo es la adaptación de la presentación realizada en el marco de las $3^{\circ}$ Jornadas sobre las Prácticas Docentes en la Universidad Pública, donde la autora disertó junto a Mariana Chaves y Elda Monetti, con la coordinación de Daniela Atairo en el PANEL 5: Docencias. Actividad desarrollada por la Dirección de Capacitación y Docencia y, la Especialización en Docencia Universitaria de la Secretaría de Asuntos Académicos de la Universidad Nacional de La Plata, en junio de 2020. Más información: https://congresos.unlp.edu.ar/jpdup/ 